Acta Crystallographica Section E

Structure Reports

Online

ISSN 1600-5368

\section{(2-Aminobenzoato- $\left.\kappa^{2} O, O^{\prime}\right)($ rac-5,5,7,- 12,12,14-hexamethyl-1,4,8,11-tetraaza- cyclotetradecane- $\left.\kappa^{4} N, N^{\prime}, N^{\prime \prime}, N^{\prime \prime \prime}\right)$ - nickel(II) perchlorate monohydrate}

\author{
Guang-Chuan $\mathrm{Ou}^{\mathrm{a}}$ and Seik Weng $\mathrm{Ng}^{\mathrm{b}}$ *
}

a Department of Biology and Chemistry, Hunan University of Science and Engineering, Yongzhou Hunan 425100, People's Republic of China, and ${ }^{\mathbf{b}}$ Department of Chemistry, University of Malaya, 50603 Kuala Lumpur, Malaysia Correspondence e-mail: seikweng@um.edu.my

Received 15 September 2010; accepted 16 September 2010

Key indicators: single-crystal X-ray study; $T=110 \mathrm{~K}$; mean $\sigma(\mathrm{C}-\mathrm{C})=0.004 \AA$; disorder in solvent or counterion; $R$ factor $=0.023 ; w R$ factor $=0.064$; data-toparameter ratio $=9.3$.

In the title salt, $\left[\mathrm{Ni}\left(\mathrm{C}_{7} \mathrm{H}_{6} \mathrm{NO}_{2}\right)\left(\mathrm{C}_{16} \mathrm{H}_{36} \mathrm{~N}_{4}\right)\right] \mathrm{ClO}_{4} \cdot \mathrm{H}_{2} \mathrm{O}$, the $\mathrm{Ni}^{\mathrm{II}}$ cation is $O, O^{\prime}$-chelated by the benzoate anion and $N, N^{\prime}, N^{\prime \prime}, N^{\prime \prime \prime}$-chelated by the macrocycle ligand, confering a distorted octahedral geometry on the metal atom. The complex cations, perchlorate anions and uncoordinated water molecules are linked by $\mathrm{N}-\mathrm{H} \cdots \mathrm{O}$ and $\mathrm{O}-\mathrm{H} \cdots \mathrm{O}$ hydrogen bonds into a three-dimensional network. The perchlorate ion is disordered over two positions in a 0.554 (8):0.446 (8) ratio.

\section{Related literature}

For two related structures, see: Ou et al. $(2008 a, b)$.

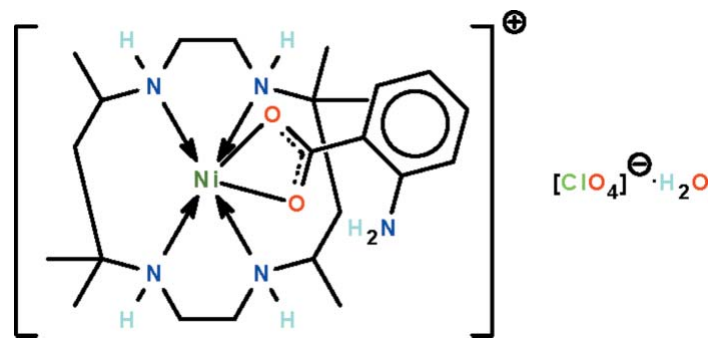

\section{Experimental}

\section{Crystal data}

\begin{tabular}{|c|c|}
\hline $\begin{array}{l}{\left[\mathrm{Ni}_{(}\left(\mathrm{C}_{7} \mathrm{H}_{6} \mathrm{NO}_{2}\right)\left(\mathrm{C}_{16} \mathrm{H}_{36} \mathrm{~N}_{4}\right)\right] \mathrm{ClO}_{4} \cdot-} \\
\quad \mathrm{H}_{2} \mathrm{O}\end{array}$ & $\begin{array}{l}\beta=90.784(1)^{\circ} \\
V=2805.5(3) \AA^{3}\end{array}$ \\
\hline$M_{r}=596.79$ & $Z=4$ \\
\hline Monoclinic, $C c$ & Mo $K \alpha$ radiation \\
\hline$a=9.6452(5) \AA$ & $\mu=0.84 \mathrm{~mm}^{-1}$ \\
\hline$b=21.5350(11) \AA$ & $T=110 \mathrm{~K}$ \\
\hline$c=13.5083(7) \AA$ & $0.45 \times 0.20 \times 0.10 \mathrm{mr}$ \\
\hline
\end{tabular}

Data collection

Bruker SMART APEX

diffractometer

Absorption correction: multi-scan (SADABS; Sheldrick, 1996)

$T_{\min }=0.818, T_{\max }=1.000$

Refinement

$R\left[F^{2}>2 \sigma\left(F^{2}\right)\right]=0.023$

$w R\left(F^{2}\right)=0.064$

$S=1.08$

3832 reflections

410 parameters

142 restraints
6638 measured reflections 3832 independent reflections 3667 reflections with $I>2 \sigma(I)$ $R_{\text {int }}=0.016$

$\mathrm{H}$ atoms treated by a mixture of independent and constrained refinement

$\Delta \rho_{\max }=0.25{\mathrm{e} \AA^{-3}}^{-3}$

$\Delta \rho_{\min }=-0.21{\mathrm{e} \AA^{-3}}^{-3}$

Absolute structure: Flack (1983), 874 Friedel pairs

Flack parameter: -0.012 (9)

Table 1

Selected bond lengths (£).

\begin{tabular}{llll}
\hline Ni1-N1 & $2.124(2)$ & Ni1-N4 & $2.087(2)$ \\
Ni1-N2 & $2.084(2)$ & Ni1-O1 & $2.1659(17)$ \\
Ni1-N3 & $2.138(2)$ & Ni1-O2 & $2.1280(16)$ \\
\hline
\end{tabular}

Table 2

Hydrogen-bond geometry $\left(\AA,^{\circ}\right)$.

\begin{tabular}{lllll}
\hline$D-\mathrm{H} \cdots A$ & $D-\mathrm{H}$ & $\mathrm{H} \cdots A$ & $D \cdots A$ & $D-\mathrm{H} \cdots A$ \\
\hline $\mathrm{N} 1-\mathrm{H} 1 \cdots \mathrm{O}^{\prime} w^{\mathrm{i}}$ & $0.86(2)$ & $2.15(2)$ & $2.995(3)$ & $166(2)$ \\
$\mathrm{N} 2-\mathrm{H} 2 \cdots 5^{\prime i i}$ & $0.86(2)$ & $2.35(2)$ & $3.125(11)$ & $150(3)$ \\
$\mathrm{N} 3-\mathrm{H} 3 \cdots \mathrm{O} 1$ & $0.86(2)$ & $2.49(3)$ & $2.885(3)$ & $109(2)$ \\
$\mathrm{N} 4-\mathrm{H} 4 \cdots 6^{\prime i i}$ & $0.86(3)$ & $2.40(2)$ & $3.195(6)$ & $155(3)$ \\
$\mathrm{N} 5-\mathrm{H} 51 \cdots \mathrm{O} 2$ & $0.85(3)$ & $2.13(3)$ & $2.751(3)$ & $129(2)$ \\
$\mathrm{N} 5-\mathrm{H} 52 \cdots 4^{\prime i}$ & $0.85(1)$ & $2.32(1)$ & $3.155(6)$ & $168(3)$ \\
$\mathrm{O} 1 \mathrm{w}-\mathrm{H} 11 \cdots \mathrm{O} 1$ & $0.84(2)$ & $1.96(2)$ & $2.795(2)$ & $172(3)$ \\
$\mathrm{O} 1 \mathrm{w}-\mathrm{H} 12 \cdots \mathrm{O}^{\mathrm{ii}}$ & $0.83(2)$ & $2.26(2)$ & $3.077(9)$ & $167(3)$ \\
$\mathrm{O} 1 \mathrm{w}-\mathrm{H} 12 \cdots \mathrm{O}^{\prime}$ & $0.83(2)$ & $2.09(2)$ & $2.890(9)$ & $160(3)$ \\
\hline
\end{tabular}

Symmetry codes: (i) $x,-y+1, z+\frac{1}{2}$; (ii) $x-\frac{1}{2},-y+\frac{1}{2}, z+\frac{1}{2}$.

Data collection: SMART (Bruker, 2003); cell refinement: SAINT (Bruker, 2003); data reduction: $S A I N T$; program(s) used to solve structure: SHELXS97 (Sheldrick, 2008); program(s) used to refine structure: SHELXL97 (Sheldrick, 2008); molecular graphics: $X$ SEED (Barbour, 2001); software used to prepare material for publication: publCIF (Westrip, 2010).

We thank the Key Subject Construction Project of Hunan Province (No. 2006-180), the Key Scientific Research Project of Hunan Provincial Education Department (No. 08 A023, 05 C736), the NSF of Hunan Province (09 J J3028) and the University of Malaya for supporting this study.

Supplementary data and figures for this paper are available from the IUCr electronic archives (Reference: XU5028).

\title{
References
}

Barbour, L. J. (2001). J. Supramol. Chem. 1, 189-191.

Bruker (2003). SAINT and SMART. Bruker AXS Inc., Madison, Wisconsin, USA.

Flack, H. D. (1983). Acta Cryst. A39, 876-881. 


\section{metal-organic compounds}

Ou, G.-C., Zhang, M. \& Yuan, X.-Y. (2008a). Acta Cryst. E64, m1010.

Ou, G.-C., Zhang, M., Yuan, X.-Y. \& Dai, Y.-Q. (2008b). Acta Cryst. E64, $\mathrm{m} 1588$.
Sheldrick, G. M. (1996). SADABS. University of Göttingen, Germany.

Sheldrick, G. M. (2008). Acta Cryst. A64, 112-122.

Westrip, S. P. (2010). J. Appl. Cryst. 43, 920-925. 


\section{supporting information}

Acta Cryst. (2010). E66, m1295-m1296 [doi:10.1107/S1600536810037116]

\section{(2-Aminobenzoato- $\left.\kappa^{2} O, O^{\prime}\right)($ rac-5,5,7,12,12,14-hexamethyl-1,4,8,11-tetraaza- cyclotetradecane- $\left.\kappa^{4} N, N^{\prime}, N^{\prime \prime}, N^{\prime \prime \prime}\right)$ nickel(II) perchlorate monohydrate}

\section{Guang-Chuan Ou and Seik Weng Ng}

\section{S1. Comment}

Previous studies on (5,5,7,12,12,14-hexamethyl-1,4,8,11-tetraazacyclotetradecane)nickel benzoate perchlorate salts have documented a chelating mode for the carboxylate ion. The perchlorate counterion is not directly involved in coordination but forms hydrogen bonds with the amino portions of the macrocycle (Ou et al., 2008a, 2008b). The title substituted benzoate has an additional amino unit that also engages in hydrogen bonding. In the salt, $\left[\mathrm{Ni}\left(\mathrm{C}_{16} \mathrm{H}_{36} \mathrm{~N}_{4}\right)\left(\mathrm{C}_{7} \mathrm{H}_{6} \mathrm{NO}_{2}\right)\right]$ $\left[\mathrm{ClO}_{4}\right] \cdot \mathrm{H}_{2} \mathrm{O}$ (Scheme I, Fig. 1), the metal atom is $O, O^{\prime}$-chelated by the carboxylate anion and $N, N^{\prime}, N^{\prime \prime}, N^{\prime \prime \prime}$-chelated by the macrocycle to confer an octahedral geometry to the metal center. The cation, anion and lattice water molecules are linked by $\mathrm{N}-\mathrm{H} \cdots \mathrm{O}$ and $\mathrm{O}-\mathrm{H} \cdots \mathrm{O}$ hydrogen bonds into a three-dimensional network.

\section{S2. Experimental}

Anthranilic acid $(0.27 \mathrm{~g}, 2 \mathrm{mmol})$ and sodium hydroxide $(0.08 \mathrm{~g}, 2 \mathrm{mmol})$ were dissolved in water $(15 \mathrm{ml})$. To this solution was added (rac-5,5,7,12,12,14-hexamethyl-1,4,8,11-tetraazacyclotetradecane)nickel diperchlorate (0.11 g, 2 mmol) dissolved in acetonitrile $(10 \mathrm{ml})$. The solution was set aside for the growth of violet crystals after a few days.

\section{S3. Refinement}

Carbon-bound $\mathrm{H}$-atoms were placed in calculated positions $(\mathrm{C}-\mathrm{H} 0.95-1.00 \AA)$ and were included in the refinement in the riding model approximation, with $U(\mathrm{H})$ set to $1.2-1.5 U(\mathrm{C})$.

The water and amino $\mathrm{H}$ atoms were located in a difference Fourier map, and were refined with distance restraints of $\mathrm{O}-$ $\mathrm{H} 0.84 \pm 0.01 \AA$ and $\mathrm{N}-\mathrm{H} 0.86 \pm 0.01 \AA$. For the water molecule, the $\mathrm{H} \cdots \mathrm{H}$ distance was restrained to $1.37 \pm 0.01 \AA$. The temperature factors were tied to those of the parent atoms by a factor of 1.2-1.5 times.

The perchlorate is disordered over two positions in a 0.554 (8):0.446 (8) ratio. All Cl-O distances were restrained to within $0.01 \AA$ of each other as were the $\mathrm{O} \cdots \mathrm{O}$ distances. The anisotropic temperature factors of the $\mathrm{O}$ atoms were restrained to be nearly isotropic. 


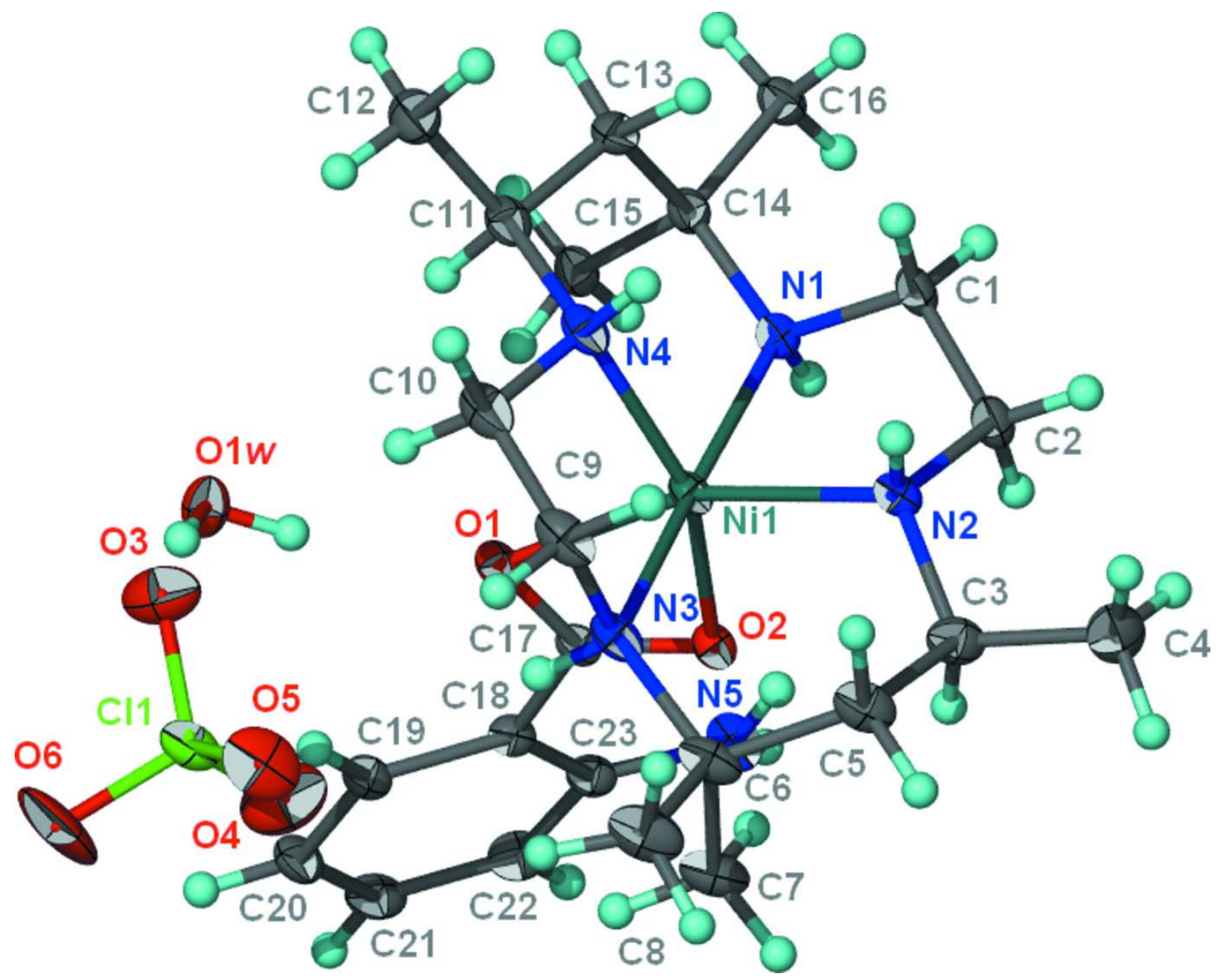

\section{Figure 1}

Thermal ellipsoid plot (Barbour, 2001) of $\left[\mathrm{Ni}\left(\mathrm{C}_{16} \mathrm{H}_{36} \mathrm{~N}_{4}\right)\left(\mathrm{C}_{7} \mathrm{H}_{6} \mathrm{NO}_{2}\right)\right]\left[\mathrm{ClO}_{4}\right] \cdot \mathrm{H}_{2} \mathrm{O}$ at the 50\% probability level; hydrogen atoms are shown as spheres of arbitrary radius. The disorder in the perchlorate is not shown.

\section{(2-Aminobenzoato- $\left.\kappa^{2} O, O^{\prime}\right)($ rac- 5,5,7,12,12,14-hexamethyl-1,4,8,11-tetraazacyclotetradecane-} $\left.\kappa^{4} N, N^{\prime}, N^{\prime \prime}, N^{\prime \prime \prime}\right)$ nickel(II) perchlorate monohydrate

Crystal data

$\left[\mathrm{Ni}\left(\mathrm{C}_{7} \mathrm{H}_{6} \mathrm{NO}_{2}\right)\left(\mathrm{C}_{16} \mathrm{H}_{36} \mathrm{~N}_{4}\right)\right] \mathrm{ClO}_{4} \cdot \mathrm{H}_{2} \mathrm{O}$

$M_{r}=596.79$

Monoclinic, $C c$

Hall symbol: $\mathrm{C}-2 \mathrm{yc}$

$a=9.6452(5) \AA$

$b=21.5350(11) \AA$

$c=13.5083$ (7) $\AA$

$\beta=90.784(1)^{\circ}$

$V=2805.5(3) \AA^{3}$

$Z=4$

\section{Data collection}

Bruker SMART APEX

diffractometer

Radiation source: fine-focus sealed tube

Graphite monochromator

$\varphi$ and $\omega$ scans

Absorption correction: multi-scan

(SADABS; Sheldrick, 1996)

$T_{\min }=0.818, T_{\max }=1.000$
$F(000)=1272$

$D_{\mathrm{x}}=1.413 \mathrm{Mg} \mathrm{m}^{-3}$

Mo $K \alpha$ radiation, $\lambda=0.71073 \AA$

Cell parameters from 5267 reflections

$\theta=2.3-27.0^{\circ}$

$\mu=0.84 \mathrm{~mm}^{-1}$

$T=110 \mathrm{~K}$

Prism, violet

$0.45 \times 0.20 \times 0.10 \mathrm{~mm}$

6638 measured reflections

3832 independent reflections

3667 reflections with $I>2 \sigma(I)$

$R_{\text {int }}=0.016$

$\theta_{\text {max }}=27.0^{\circ}, \theta_{\min }=1.9^{\circ}$

$h=-11 \rightarrow 12$

$k=-26 \rightarrow 26$

$l=-8 \rightarrow 17$ 


\section{Refinement}

Refinement on $F^{2}$

Least-squares matrix: full

$R\left[F^{2}>2 \sigma\left(F^{2}\right)\right]=0.023$

$w R\left(F^{2}\right)=0.064$

$S=1.08$

3832 reflections

410 parameters

142 restraints

Primary atom site location: structure-invariant direct methods

Secondary atom site location: difference Fourier map
Hydrogen site location: inferred from neighbouring sites

$\mathrm{H}$ atoms treated by a mixture of independent and constrained refinement

$w=1 /\left[\sigma^{2}\left(F_{\mathrm{o}}^{2}\right)+(0.0373 P)^{2}+0.1275 P\right]$ where $P=\left(F_{\mathrm{o}}^{2}+2 F_{\mathrm{c}}^{2}\right) / 3$

$(\Delta / \sigma)_{\max }=0.001$

$\Delta \rho_{\max }=0.25$ e $\AA^{-3}$

$\Delta \rho_{\text {min }}=-0.21$ e $\AA^{-3}$

Absolute structure: Flack (1983), 874 Friedel pairs

Absolute structure parameter: -0.012 (9)

Fractional atomic coordinates and isotropic or equivalent isotropic displacement parameters $\left(\AA^{2}\right)$

\begin{tabular}{|c|c|c|c|c|c|}
\hline & $x$ & $y$ & $z$ & $U_{\text {iso }} * / U_{\text {eq }}$ & Occ. $(<1)$ \\
\hline Nil & 0.50011 & $0.399116(12)$ & $0.49992(3)$ & $0.01859(8)$ & \\
\hline $\mathrm{O} 1$ & $0.58175(16)$ & $0.44546(8)$ & $0.37089(12)$ & $0.0219(4)$ & \\
\hline $\mathrm{O} 2$ & $0.66792(16)$ & $0.46225(7)$ & $0.51979(12)$ & 0.0201 & \\
\hline $\mathrm{Cl1}$ & $0.6956(5)$ & $0.2675(2)$ & 0.1539 & $0.0295(7)$ & $0.554(8)$ \\
\hline $\mathrm{O} 3$ & $0.5575(5)$ & $0.2886(4)$ & $0.1347(6)$ & 0.0444 (19) & $0.554(8)$ \\
\hline $\mathrm{O} 4$ & $0.7620(5)$ & $0.3111(2)$ & $0.2197(4)$ & $0.0581(17)$ & $0.554(8)$ \\
\hline $\mathrm{O} 5$ & $0.6945(9)$ & $0.2080(2)$ & $0.2001(5)$ & 0.0439 (19) & $0.554(8)$ \\
\hline O6 & $0.7708(7)$ & $0.2650(3)$ & $0.0641(4)$ & $0.070(2)$ & $0.554(8)$ \\
\hline $\mathrm{C}^{\prime \prime} 1^{\prime}$ & $0.6956(6)$ & $0.2641(3)$ & $0.1262(4)$ & $0.0311(10)$ & $0.446(8)$ \\
\hline O3' & $0.5794(8)$ & $0.2974(4)$ & $0.1623(6)$ & $0.042(2)$ & $0.446(8)$ \\
\hline $\mathrm{O} 4^{\prime}$ & $0.8213(6)$ & $0.2957(3)$ & $0.1496(6)$ & $0.060(3)$ & $0.446(8)$ \\
\hline O5' & $0.6992(12)$ & 0.2028 & $0.1656(9)$ & $0.057(3)$ & $0.446(8)$ \\
\hline O6 $6^{\prime}$ & $0.6838(8)$ & $0.2577(3)$ & $0.0218(4)$ & $0.058(2)$ & $0.446(8)$ \\
\hline $\mathrm{O} 1 \mathrm{~W}$ & $0.5173(2)$ & $0.42855(9)$ & $0.17034(14)$ & $0.0296(4)$ & \\
\hline H11 & $0.545(3)$ & $0.4343(13)$ & $0.2291(11)$ & $0.044^{*}$ & \\
\hline H12 & $0.537(4)$ & $0.3925(8)$ & $0.153(2)$ & $0.044 *$ & \\
\hline N1 & $0.3714(2)$ & $0.46844(10)$ & $0.56243(16)$ & $0.0190(4)$ & \\
\hline H1 & $0.423(2)$ & $0.4982(10)$ & $0.585(2)$ & $0.023^{*}$ & \\
\hline $\mathrm{N} 2$ & 0.4909 (2) & $0.35763(9)$ & $0.63905(16)$ & $0.0211(4)$ & \\
\hline $\mathrm{H} 2$ & $0.428(2)$ & $0.3296(10)$ & $0.636(2)$ & $0.025 *$ & \\
\hline N3 & $0.6127(2)$ & $0.32080(10)$ & $0.44631(17)$ & 0.0243 & \\
\hline H3 & $0.652(3)$ & $0.3351(13)$ & $0.3944(14)$ & $0.029 *$ & \\
\hline N4 & $0.3296(2)$ & $0.36063(10)$ & $0.42555(16)$ & $0.0242(4)$ & \\
\hline $\mathrm{H} 4$ & $0.280(3)$ & $0.3380(12)$ & $0.4632(19)$ & $0.029 *$ & \\
\hline N5 & $0.7978(2)$ & $0.57537(11)$ & $0.53966(18)$ & $0.0289(5)$ & \\
\hline H51 & $0.739(3)$ & $0.5514(12)$ & $0.567(2)$ & $0.035^{*}$ & \\
\hline H52 & $0.814(3)$ & $0.6115(8)$ & $0.563(2)$ & $0.035^{*}$ & \\
\hline $\mathrm{C} 1$ & $0.3207(3)$ & $0.44029(12)$ & $0.65532(18)$ & $0.0245(5)$ & \\
\hline $\mathrm{H} 1 \mathrm{~A}$ & 0.2438 & 0.4112 & 0.6402 & $0.029 *$ & \\
\hline H1B & 0.2852 & 0.4732 & 0.6995 & $0.029 *$ & \\
\hline $\mathrm{C} 2$ & $0.4374(3)$ & $0.40601(11)$ & $0.70620(19)$ & $0.0253(5)$ & \\
\hline $\mathrm{H} 2 \mathrm{~A}$ & 0.5126 & 0.4354 & 0.7241 & $0.030 *$ & \\
\hline
\end{tabular}




\begin{tabular}{|c|c|c|c|c|}
\hline $\mathrm{H} 2 \mathrm{~B}$ & 0.4037 & 0.3866 & 0.7678 & $0.030^{*}$ \\
\hline $\mathrm{C} 3$ & $0.6234(3)$ & $0.33020(13)$ & $0.6779(2)$ & $0.0282(6)$ \\
\hline $\mathrm{H} 3 \mathrm{a}$ & 0.6968 & 0.3629 & 0.6751 & $0.034^{*}$ \\
\hline $\mathrm{C} 4$ & $0.6109(3)$ & $0.30893(15)$ & $0.7856(2)$ & $0.0378(7)$ \\
\hline $\mathrm{H} 4 \mathrm{~A}$ & 0.5814 & 0.3440 & 0.8265 & $0.057^{*}$ \\
\hline $\mathrm{H} 4 \mathrm{~B}$ & 0.7011 & 0.2937 & 0.8096 & $0.057^{*}$ \\
\hline $\mathrm{H} 4 \mathrm{C}$ & 0.5423 & 0.2755 & 0.7895 & $0.057^{*}$ \\
\hline $\mathrm{C} 5$ & 0.6684 & $0.27612(12)$ & $0.6133(2)$ & $0.0293(6)$ \\
\hline $\mathrm{H} 5 \mathrm{~A}$ & 0.5883 & 0.2477 & 0.6059 & $0.035^{*}$ \\
\hline $\mathrm{H} 5 \mathrm{~B}$ & 0.7418 & 0.2532 & 0.6499 & $0.035^{*}$ \\
\hline C6 & $0.7230(3)$ & $0.28992(12)$ & $0.5088(2)$ & $0.0298(6)$ \\
\hline $\mathrm{C} 7$ & 0.8464 & $0.33447(13)$ & $0.5146(2)$ & $0.0353(7)$ \\
\hline $\mathrm{H} 7 \mathrm{~A}$ & 0.8832 & 0.3409 & 0.4481 & $0.053^{*}$ \\
\hline H7B & 0.9188 & 0.3167 & 0.5575 & $0.053^{*}$ \\
\hline $\mathrm{H} 7 \mathrm{C}$ & 0.8162 & 0.3743 & 0.5418 & $0.053 *$ \\
\hline $\mathrm{C} 8$ & $0.7727(3)$ & $0.22831(14)$ & $0.4626(3)$ & $0.0393(7)$ \\
\hline H8A & 0.7921 & 0.2348 & 0.3923 & $0.059^{*}$ \\
\hline H8B & 0.7003 & 0.1967 & 0.4692 & $0.059^{*}$ \\
\hline $\mathrm{H} 8 \mathrm{C}$ & 0.8573 & 0.2143 & 0.4969 & $0.059^{*}$ \\
\hline C9 & $0.5037(3)$ & $0.27846(12)$ & $0.4086(2)$ & $0.0297(6)$ \\
\hline H9A & 0.4632 & 0.2551 & 0.4643 & $0.036^{*}$ \\
\hline H9B & 0.5444 & 0.2483 & 0.3620 & $0.036^{*}$ \\
\hline $\mathrm{C} 10$ & $0.3920(3)$ & $0.31573(13)$ & $0.3564(2)$ & $0.0288(6)$ \\
\hline $\mathrm{H} 10 \mathrm{~A}$ & 0.4324 & 0.3383 & 0.2998 & $0.035^{*}$ \\
\hline $\mathrm{H} 10 \mathrm{~B}$ & 0.3194 & 0.2874 & 0.3304 & $0.035^{*}$ \\
\hline $\mathrm{C} 11$ & $0.2384(3)$ & $0.40671(11)$ & $0.37461(19)$ & $0.0241(5)$ \\
\hline H11A & 0.2979 & 0.4328 & 0.3310 & $0.029^{*}$ \\
\hline $\mathrm{C} 12$ & $0.1271(3)$ & $0.37581(15)$ & $0.3090(2)$ & $0.0335(6)$ \\
\hline $\mathrm{H} 12 \mathrm{~A}$ & 0.1719 & 0.3517 & 0.2568 & $0.050^{*}$ \\
\hline H12B & 0.0680 & 0.4078 & 0.2788 & $0.050^{*}$ \\
\hline $\mathrm{H} 12 \mathrm{C}$ & 0.0704 & 0.3481 & 0.3494 & $0.050^{*}$ \\
\hline $\mathrm{C} 13$ & $0.1684(2)$ & $0.45014(12)$ & 0.44934 (19) & $0.0238(5)$ \\
\hline H13A & 0.1253 & 0.4240 & 0.5007 & $0.029 *$ \\
\hline H13B & 0.0923 & 0.4722 & 0.4142 & $0.029 *$ \\
\hline $\mathrm{C} 14$ & $0.2594(2)$ & $0.49930(12)$ & $0.50235(18)$ & $0.0217(5)$ \\
\hline $\mathrm{C} 15$ & $0.3297(3)$ & $0.54169(12)$ & 0.42814 (19) & $0.0237(5)$ \\
\hline H15A & 0.3819 & 0.5740 & 0.4637 & $0.036^{*}$ \\
\hline H15B & 0.2592 & 0.5611 & 0.3853 & $0.036^{*}$ \\
\hline $\mathrm{H} 15 \mathrm{C}$ & 0.3932 & 0.5173 & 0.3876 & $0.036^{*}$ \\
\hline $\mathrm{C} 16$ & $0.1671(3)$ & $0.54025(13)$ & $0.5675(2)$ & $0.0259(6)$ \\
\hline H16A & 0.2256 & 0.5678 & 0.6079 & $0.039^{*}$ \\
\hline H16B & 0.1117 & 0.5138 & 0.6109 & $0.039 *$ \\
\hline $\mathrm{H} 16 \mathrm{C}$ & 0.1053 & 0.5652 & 0.5253 & $0.039 *$ \\
\hline $\mathrm{C} 17$ & $0.6690(2)$ & $0.47306(11)$ & $0.42754(19)$ & $0.0186(5)$ \\
\hline $\mathrm{C} 18$ & $0.7701(2)$ & $0.51743(11)$ & $0.38477(18)$ & $0.0198(5)$ \\
\hline C19 & $0.8068(2)$ & $0.51078(12)$ & $0.28554(19)$ & $0.0234(5)$ \\
\hline H19 & 0.7678 & 0.4777 & 0.2479 & $0.028^{*}$ \\
\hline $\mathrm{C} 20$ & $0.8984(3)$ & $0.55104(13)$ & $0.2407(2)$ & $0.0292(6)$ \\
\hline
\end{tabular}




\begin{tabular}{lllll}
$\mathrm{H} 20$ & 0.9247 & 0.5450 & 0.1739 & $0.035^{*}$ \\
$\mathrm{C} 21$ & $0.9514(3)$ & $0.60087(12)$ & $0.2959(2)$ & $0.0301(6)$ \\
$\mathrm{H} 21$ & 1.0125 & 0.6296 & 0.2657 & $0.036^{*}$ \\
$\mathrm{C} 22$ & $0.9161(3)$ & $0.60871(12)$ & $0.3934(2)$ & $0.0276(6)$ \\
$\mathrm{H} 22$ & 0.9532 & 0.6430 & 0.4292 & $0.033^{*}$ \\
$\mathrm{C} 23$ & $0.8259(2)$ & $0.56699(12)$ & $0.4417(2)$ & $0.0219(5)$ \\
\hline
\end{tabular}

Atomic displacement parameters $\left(\AA^{2}\right)$

\begin{tabular}{|c|c|c|c|c|c|c|}
\hline & $U^{11}$ & $U^{22}$ & $U^{33}$ & $U^{12}$ & $U^{13}$ & $U^{23}$ \\
\hline Nil & $0.01972(13)$ & 0.01555 (14) & $0.02064(13)$ & $-0.00292(12)$ & $0.00605(9)$ & -0.00109 \\
\hline $\mathrm{O} 1$ & $0.0225(8)$ & $0.0217(9)$ & $0.0217(8)$ & $-0.0068(7)$ & $0.0050(7)$ & $-0.0017(7)$ \\
\hline $\mathrm{O} 2$ & $0.0227(8)$ & $0.0171(9)$ & $0.0207(8)$ & $-0.0015(6)$ & $0.0043(7)$ & $-0.0002(7)$ \\
\hline $\mathrm{Cl1}$ & $0.0273(9)$ & $0.0245(10)$ & $0.0371(17)$ & $0.0031(6)$ & $0.0107(11)$ & $0.0046(11)$ \\
\hline $\mathrm{O} 3$ & $0.036(2)$ & $0.035(3)$ & $0.062(4)$ & $0.005(2)$ & $-0.007(2)$ & $-0.016(3)$ \\
\hline $\mathrm{O} 4$ & $0.053(3)$ & $0.045(3)$ & $0.075(4)$ & $-0.004(2)$ & $-0.017(3)$ & $-0.008(2)$ \\
\hline O5 & $0.055(3)$ & $0.015(2)$ & $0.062(4)$ & $0.000(2)$ & $0.014(3)$ & $0.010(2)$ \\
\hline O6 & $0.071(4)$ & $0.077(4)$ & 0.065 & $0.031(3)$ & $0.052(4)$ & $0.012(3)$ \\
\hline $\mathrm{Cl1}^{\prime}$ & $0.0308(12)$ & $0.0213(12)$ & $0.041(3)$ & $0.0025(8)$ & $0.0045(16)$ & $0.0042(15)$ \\
\hline O3' & $0.048(4)$ & $0.033(4)$ & $0.047(4)$ & $0.016(3)$ & $0.024(3)$ & $0.001(3)$ \\
\hline O4' & $0.055(3)$ & $0.038(3)$ & $0.087(6)$ & $-0.014(3)$ & $-0.021(3)$ & $0.020(3)$ \\
\hline O5' & $0.051(4)$ & $0.027(4)$ & $0.094(7)$ & $0.008(3)$ & $0.017(5)$ & $0.010(4)$ \\
\hline $\mathrm{O}^{\prime}$ & $0.055(4)$ & $0.079(4)$ & $0.041(3)$ & $0.016(3)$ & 0.009 (3) & $-0.017(3)$ \\
\hline $\mathrm{O} 1 \mathrm{~W}$ & $0.0405(11)$ & $0.0243(10)$ & $0.0239(9)$ & $0.0039(8)$ & $-0.0026(8)$ & $0.0000(7)$ \\
\hline N1 & $0.0222(10)$ & $0.0145(10)$ & $0.0205(10)$ & $-0.0003(8)$ & $0.0027(8)$ & $0.0008(8)$ \\
\hline $\mathrm{N} 2$ & $0.0208(10)$ & $0.0180(10)$ & $0.0248(10)$ & $0.0008(8)$ & $0.0057(8)$ & $0.0009(8)$ \\
\hline N3 & $0.0254(10)$ & $0.0186(11)$ & $0.0291(11)$ & $-0.0042(8)$ & $0.0107(9)$ & $-0.0014(9)$ \\
\hline N4 & $0.0258(10)$ & $0.0228(11)$ & $0.0242(10)$ & $-0.0061(8)$ & $0.0086(8)$ & $-0.0022(9)$ \\
\hline N5 & $0.0337(12)$ & $0.0215(11)$ & $0.0315(12)$ & $-0.0095(9)$ & $0.0063(9)$ & $-0.0075(9)$ \\
\hline $\mathrm{C} 1$ & $0.0287(12)$ & $0.0262(13)$ & $0.0187(11)$ & $0.0058(10)$ & $0.0078(10)$ & $0.0013(10)$ \\
\hline $\mathrm{C} 2$ & $0.0314(13)$ & $0.0246(13)$ & $0.0202(11)$ & $0.0062(10)$ & $0.0056(10)$ & $0.0018(10)$ \\
\hline $\mathrm{C} 3$ & $0.0250(12)$ & $0.0240(14)$ & $0.0356(15)$ & $0.0034(10)$ & $0.0030(11)$ & $0.0032(11)$ \\
\hline $\mathrm{C} 4$ & $0.0416(16)$ & $0.0366(16)$ & $0.0353(15)$ & $0.0169(13)$ & $0.0014(13)$ & $0.0070(13)$ \\
\hline $\mathrm{C} 5$ & $0.0295(13)$ & $0.0180(12)$ & $0.0406(15)$ & $0.0043(10)$ & $0.0081(11)$ & $0.0038(11)$ \\
\hline C6 & $0.0245(13)$ & $0.0232(14)$ & $0.0420(16)$ & $0.0016(10)$ & $0.0122(11)$ & $-0.0024(12)$ \\
\hline $\mathrm{C} 7$ & $0.0229(13)$ & $0.0326(16)$ & $0.0507(17)$ & $0.0009(11)$ & $0.0090(12)$ & $-0.0002(13)$ \\
\hline $\mathrm{C} 8$ & $0.0370(16)$ & $0.0235(15)$ & $0.058(2)$ & $0.0074(12)$ & $0.0127(15)$ & $-0.0063(14)$ \\
\hline C9 & $0.0347(13)$ & $0.0181(13)$ & $0.0368(14)$ & $-0.0057(10)$ & $0.0146(11)$ & $-0.0073(11)$ \\
\hline $\mathrm{C} 10$ & $0.0315(14)$ & $0.0250(14)$ & $0.0301(14)$ & $-0.0081(10)$ & $0.0079(11)$ & $-0.0125(11)$ \\
\hline C11 & $0.0247(12)$ & $0.0247(13)$ & $0.0232(12)$ & $-0.0063(9)$ & $0.0049(10)$ & $-0.0010(10)$ \\
\hline $\mathrm{C} 12$ & $0.0295(13)$ & $0.0429(17)$ & $0.0282(13)$ & $-0.0081(12)$ & $0.0010(11)$ & $-0.0065(12)$ \\
\hline $\mathrm{C} 13$ & $0.0193(11)$ & $0.0276(13)$ & $0.0245(12)$ & $-0.0013(9)$ & $0.0031(10)$ & $0.0017(10)$ \\
\hline $\mathrm{C} 14$ & $0.0229(11)$ & $0.0224(12)$ & $0.0200(11)$ & $0.0015(9)$ & $0.0040(9)$ & $0.0031(10)$ \\
\hline $\mathrm{C} 15$ & $0.0278(12)$ & $0.0222(12)$ & $0.0212(11)$ & $0.0021(9)$ & $0.0048(10)$ & $0.0034(10)$ \\
\hline $\mathrm{C} 16$ & $0.0263(13)$ & $0.0259(15)$ & $0.0256(13)$ & $0.0059(10)$ & $0.0062(10)$ & $0.0040(11)$ \\
\hline $\mathrm{C} 17$ & $0.0197(11)$ & $0.0140(11)$ & $0.0222(12)$ & $0.0024(9)$ & $0.0045(9)$ & $-0.0008(9)$ \\
\hline $\mathrm{C} 18$ & $0.0190(11)$ & $0.0170(12)$ & $0.0236(12)$ & $0.0000(8)$ & $0.0017(9)$ & $0.0014(9)$ \\
\hline C19 & $0.0210(12)$ & $0.0238(13)$ & $0.0255(12)$ & $-0.0011(9)$ & $0.0037(10)$ & $0.0014(10)$ \\
\hline
\end{tabular}


supporting information

\begin{tabular}{lllllll}
$\mathrm{C} 20$ & $0.0253(12)$ & $0.0355(15)$ & $0.0269(13)$ & $0.0006(10)$ & $0.0083(10)$ & $0.0080(11)$ \\
$\mathrm{C} 21$ & $0.0210(12)$ & $0.0288(14)$ & $0.0405(15)$ & $-0.0034(9)$ & $0.0045(11)$ & $0.0131(12)$ \\
$\mathrm{C} 22$ & $0.0249(12)$ & $0.0199(13)$ & $0.0378(15)$ & $-0.0048(9)$ & $-0.0005(11)$ & $0.0034(11)$ \\
$\mathrm{C} 23$ & $0.0194(11)$ & $0.0193(12)$ & $0.0269(12)$ & $0.0015(9)$ & $0.0022(10)$ & $0.0017(10)$ \\
\hline
\end{tabular}

Geometric parameters $\left(\AA,{ }^{\circ}\right)$

\begin{tabular}{|c|c|c|c|}
\hline $\mathrm{Ni} 1-\mathrm{N} 1$ & $2.124(2)$ & $\mathrm{C} 5-\mathrm{C} 6$ & $1.541(4)$ \\
\hline $\mathrm{Ni} 1-\mathrm{N} 2$ & $2.084(2)$ & $\mathrm{C} 5-\mathrm{H} 5 \mathrm{~A}$ & 0.9900 \\
\hline $\mathrm{Ni1}-\mathrm{N} 3$ & $2.138(2)$ & $\mathrm{C} 5-\mathrm{H} 5 \mathrm{~B}$ & 0.9900 \\
\hline $\mathrm{Ni} 1-\mathrm{N} 4$ & $2.087(2)$ & $\mathrm{C} 6-\mathrm{C} 7$ & $1.530(4)$ \\
\hline $\mathrm{Ni} 1-\mathrm{O} 1$ & $2.1659(17)$ & $\mathrm{C} 6-\mathrm{C} 8$ & $1.546(4)$ \\
\hline $\mathrm{Ni} 1-\mathrm{O} 2$ & $2.1280(16)$ & $\mathrm{C} 7-\mathrm{H} 7 \mathrm{~A}$ & 0.9800 \\
\hline $\mathrm{O} 1-\mathrm{C} 17$ & $1.276(3)$ & $\mathrm{C} 7-\mathrm{H} 7 \mathrm{~B}$ & 0.9800 \\
\hline $\mathrm{O} 2-\mathrm{C} 17$ & $1.268(3)$ & $\mathrm{C} 7-\mathrm{H} 7 \mathrm{C}$ & 0.9800 \\
\hline $\mathrm{Cl1}-\mathrm{O} 6$ & $1.423(4)$ & $\mathrm{C} 8-\mathrm{H} 8 \mathrm{~A}$ & 0.9800 \\
\hline $\mathrm{Cl1}-\mathrm{O} 5$ & $1.425(5)$ & $\mathrm{C} 8-\mathrm{H} 8 \mathrm{~B}$ & 0.9800 \\
\hline $\mathrm{Cl1}-\mathrm{O} 3$ & $1.427(5)$ & $\mathrm{C} 8-\mathrm{H} 8 \mathrm{C}$ & 0.9800 \\
\hline $\mathrm{Cl1}-\mathrm{O} 4$ & $1.437(5)$ & $\mathrm{C} 9-\mathrm{C} 10$ & $1.510(4)$ \\
\hline $\mathrm{Cl1}^{\prime}-\mathrm{O} 6^{\prime}$ & $1.421(5)$ & C9-H9A & 0.9900 \\
\hline $\mathrm{Cl1}^{\prime}-\mathrm{O}^{\prime}$ & $1.423(6)$ & C9-H9B & 0.9900 \\
\hline $\mathrm{Cl1}^{\prime}-\mathrm{O} 3^{\prime}$ & $1.422(5)$ & $\mathrm{C} 10-\mathrm{H} 10 \mathrm{~A}$ & 0.9900 \\
\hline $\mathrm{Cl1}^{\prime}-\mathrm{O} 4^{\prime}$ & $1.422(6)$ & $\mathrm{C} 10-\mathrm{H} 10 \mathrm{~B}$ & 0.9900 \\
\hline $\mathrm{O} 1 \mathrm{~W}-\mathrm{H} 11$ & $0.843(10)$ & $\mathrm{C} 11-\mathrm{C} 12$ & $1.534(4)$ \\
\hline $\mathrm{O} 1 \mathrm{~W}-\mathrm{H} 12$ & $0.833(10)$ & $\mathrm{C} 11-\mathrm{C} 13$ & $1.539(3)$ \\
\hline $\mathrm{N} 1-\mathrm{C} 1$ & $1.482(3)$ & $\mathrm{C} 11-\mathrm{H} 11 \mathrm{~A}$ & 1.0000 \\
\hline $\mathrm{N} 1-\mathrm{C} 14$ & $1.498(3)$ & $\mathrm{C} 12-\mathrm{H} 12 \mathrm{~A}$ & 0.9800 \\
\hline $\mathrm{N} 1-\mathrm{H} 1$ & $0.861(10)$ & $\mathrm{C} 12-\mathrm{H} 12 \mathrm{~B}$ & 0.9800 \\
\hline $\mathrm{N} 2-\mathrm{C} 2$ & $1.479(3)$ & $\mathrm{C} 12-\mathrm{H} 12 \mathrm{C}$ & 0.9800 \\
\hline $\mathrm{N} 2-\mathrm{C} 3$ & $1.496(3)$ & $\mathrm{C} 13-\mathrm{C} 14$ & $1.545(3)$ \\
\hline $\mathrm{N} 2-\mathrm{H} 2$ & $0.858(10)$ & $\mathrm{C} 13-\mathrm{H} 13 \mathrm{~A}$ & 0.9900 \\
\hline $\mathrm{N} 3-\mathrm{C} 9$ & $1.477(3)$ & C13-H13B & 0.9900 \\
\hline $\mathrm{N} 3-\mathrm{C} 6$ & $1.504(4)$ & $\mathrm{C} 14-\mathrm{C} 15$ & $1.522(3)$ \\
\hline N3-H3 & $0.860(10)$ & $\mathrm{C} 14-\mathrm{C} 16$ & $1.538(3)$ \\
\hline $\mathrm{N} 4-\mathrm{C} 10$ & $1.478(3)$ & $\mathrm{C} 15-\mathrm{H} 15 \mathrm{~A}$ & 0.9800 \\
\hline $\mathrm{N} 4-\mathrm{C} 11$ & $1.489(3)$ & $\mathrm{C} 15-\mathrm{H} 15 \mathrm{~B}$ & 0.9800 \\
\hline $\mathrm{N} 4-\mathrm{H} 4$ & $0.86(3)$ & $\mathrm{C} 15-\mathrm{H} 15 \mathrm{C}$ & 0.9800 \\
\hline $\mathrm{N} 5-\mathrm{C} 23$ & $1.366(3)$ & $\mathrm{C} 16-\mathrm{H} 16 \mathrm{~A}$ & 0.9800 \\
\hline N5-H51 & $0.86(3)$ & $\mathrm{C} 16-\mathrm{H} 16 \mathrm{~B}$ & 0.9800 \\
\hline N5-H52 & $0.854(10)$ & $\mathrm{C} 16-\mathrm{H} 16 \mathrm{C}$ & 0.9800 \\
\hline $\mathrm{C} 1-\mathrm{C} 2$ & $1.504(3)$ & $\mathrm{C} 17-\mathrm{C} 18$ & $1.487(3)$ \\
\hline $\mathrm{C} 1-\mathrm{H} 1 \mathrm{~A}$ & 0.9900 & $\mathrm{C} 18-\mathrm{C} 19$ & $1.399(3)$ \\
\hline $\mathrm{C} 1-\mathrm{H} 1 \mathrm{~B}$ & 0.9900 & $\mathrm{C} 18-\mathrm{C} 23$ & $1.418(3)$ \\
\hline $\mathrm{C} 2-\mathrm{H} 2 \mathrm{~A}$ & 0.9900 & $\mathrm{C} 19-\mathrm{C} 20$ & $1.383(3)$ \\
\hline $\mathrm{C} 2-\mathrm{H} 2 \mathrm{~B}$ & 0.9900 & C19-H19 & 0.9500 \\
\hline $\mathrm{C} 3-\mathrm{C} 5$ & $1.522(4)$ & $\mathrm{C} 20-\mathrm{C} 21$ & $1.400(4)$ \\
\hline $\mathrm{C} 3-\mathrm{C} 4$ & $1.532(4)$ & $\mathrm{C} 20-\mathrm{H} 20$ & 0.9500 \\
\hline $\mathrm{C} 3-\mathrm{H} 3 \mathrm{a}$ & 1.0000 & $\mathrm{C} 21-\mathrm{C} 22$ & $1.375(4)$ \\
\hline
\end{tabular}




\begin{tabular}{|c|c|c|c|}
\hline $\mathrm{C} 4-\mathrm{H} 4 \mathrm{~A}$ & 0.9800 & $\mathrm{C} 21-\mathrm{H} 21$ & 0.9500 \\
\hline $\mathrm{C} 4-\mathrm{H} 4 \mathrm{~B}$ & 0.9800 & $\mathrm{C} 22-\mathrm{C} 23$ & $1.416(4)$ \\
\hline $\mathrm{C} 4-\mathrm{H} 4 \mathrm{C}$ & 0.9800 & $\mathrm{C} 22-\mathrm{H} 22$ & 0.9500 \\
\hline $\mathrm{N} 2-\mathrm{Ni1}-\mathrm{N} 4$ & $102.76(8)$ & $\mathrm{N} 3-\mathrm{C} 6-\mathrm{C} 7$ & $107.2(2)$ \\
\hline $\mathrm{N} 2-\mathrm{Ni} 1-\mathrm{N} 1$ & $84.86(8)$ & $\mathrm{N} 3-\mathrm{C} 6-\mathrm{C} 5$ & $110.60(19)$ \\
\hline $\mathrm{N} 4-\mathrm{Ni1}-\mathrm{N} 1$ & $90.54(8)$ & $\mathrm{C} 7-\mathrm{C} 6-\mathrm{C} 5$ & $110.5(2)$ \\
\hline $\mathrm{N} 2-\mathrm{Ni} 1-\mathrm{O} 2$ & $101.64(7)$ & $\mathrm{N} 3-\mathrm{C} 6-\mathrm{C} 8$ & $111.9(2)$ \\
\hline $\mathrm{N} 4-\mathrm{Ni1}-\mathrm{O} 2$ & $155.16(8)$ & $\mathrm{C} 7-\mathrm{C} 6-\mathrm{C} 8$ & $108.2(2)$ \\
\hline $\mathrm{N} 1-\mathrm{Ni} 1-\mathrm{O} 2$ & $87.05(7)$ & $\mathrm{C} 5-\mathrm{C} 6-\mathrm{C} 8$ & $108.4(2)$ \\
\hline $\mathrm{N} 2-\mathrm{Ni} 1-\mathrm{N} 3$ & $89.73(8)$ & $\mathrm{C} 6-\mathrm{C} 7-\mathrm{H} 7 \mathrm{~A}$ & 109.5 \\
\hline $\mathrm{N} 4-\mathrm{Ni1}-\mathrm{N} 3$ & $85.66(8)$ & $\mathrm{C} 6-\mathrm{C} 7-\mathrm{H} 7 \mathrm{~B}$ & 109.5 \\
\hline $\mathrm{N} 1-\mathrm{Ni1}-\mathrm{N} 3$ & $172.56(8)$ & $\mathrm{H} 7 \mathrm{~A}-\mathrm{C} 7-\mathrm{H} 7 \mathrm{~B}$ & 109.5 \\
\hline $\mathrm{O} 2-\mathrm{Ni} 1-\mathrm{N} 3$ & $99.07(7)$ & $\mathrm{C} 6-\mathrm{C} 7-\mathrm{H} 7 \mathrm{C}$ & 109.5 \\
\hline $\mathrm{N} 2-\mathrm{Ni} 1-\mathrm{O} 1$ & $160.67(7)$ & $\mathrm{H} 7 \mathrm{~A}-\mathrm{C} 7-\mathrm{H} 7 \mathrm{C}$ & 109.5 \\
\hline $\mathrm{N} 4-\mathrm{Ni1}-\mathrm{O} 1$ & $95.07(7)$ & $\mathrm{H} 7 \mathrm{~B}-\mathrm{C} 7-\mathrm{H} 7 \mathrm{C}$ & 109.5 \\
\hline $\mathrm{N} 1-\mathrm{Ni1}-\mathrm{O} 1$ & $102.53(7)$ & $\mathrm{C} 6-\mathrm{C} 8-\mathrm{H} 8 \mathrm{~A}$ & 109.5 \\
\hline $\mathrm{O} 2-\mathrm{Ni} 1-\mathrm{O} 1$ & $61.50(6)$ & $\mathrm{C} 6-\mathrm{C} 8-\mathrm{H} 8 \mathrm{~B}$ & 109.5 \\
\hline N3-Ni1-O1 & $84.20(7)$ & $\mathrm{H} 8 \mathrm{~A}-\mathrm{C} 8-\mathrm{H} 8 \mathrm{~B}$ & 109.5 \\
\hline $\mathrm{C} 17-\mathrm{O} 1-\mathrm{Ni1}$ & $88.63(14)$ & $\mathrm{C} 6-\mathrm{C} 8-\mathrm{H} 8 \mathrm{C}$ & 109.5 \\
\hline $\mathrm{C} 17-\mathrm{O} 2-\mathrm{Ni} 1$ & $90.56(14)$ & $\mathrm{H} 8 \mathrm{~A}-\mathrm{C} 8-\mathrm{H} 8 \mathrm{C}$ & 109.5 \\
\hline $\mathrm{O} 6-\mathrm{C} 11-\mathrm{O} 5$ & $110.2(4)$ & $\mathrm{H} 8 \mathrm{~B}-\mathrm{C} 8-\mathrm{H} 8 \mathrm{C}$ & 109.5 \\
\hline $\mathrm{O} 6-\mathrm{Cl1}-\mathrm{O} 3$ & $110.1(4)$ & $\mathrm{N} 3-\mathrm{C} 9-\mathrm{C} 10$ & $109.4(2)$ \\
\hline $\mathrm{O} 5-\mathrm{Cl1}-\mathrm{O} 3$ & $110.7(4)$ & $\mathrm{N} 3-\mathrm{C} 9-\mathrm{H} 9 \mathrm{~A}$ & 109.8 \\
\hline $\mathrm{O} 6-\mathrm{Cl1}-\mathrm{O} 4$ & $108.9(4)$ & $\mathrm{C} 10-\mathrm{C} 9-\mathrm{H} 9 \mathrm{~A}$ & 109.8 \\
\hline $\mathrm{O} 5-\mathrm{Cl1}-\mathrm{O} 4$ & $108.8(4)$ & $\mathrm{N} 3-\mathrm{C} 9-\mathrm{H} 9 \mathrm{~B}$ & 109.8 \\
\hline $\mathrm{O} 3-\mathrm{Cl} 1-\mathrm{O} 4$ & $108.1(4)$ & $\mathrm{C} 10-\mathrm{C} 9-\mathrm{H} 9 \mathrm{~B}$ & 109.8 \\
\hline $\mathrm{O} 6^{\prime}-\mathrm{Cl1}^{\prime}-\mathrm{O} 5^{\prime}$ & $106.4(7)$ & $\mathrm{H} 9 \mathrm{~A}-\mathrm{C} 9-\mathrm{H} 9 \mathrm{~B}$ & 108.2 \\
\hline $\mathrm{O} 6^{\prime}-\mathrm{Cl1}^{\prime}-\mathrm{O} 3^{\prime}$ & $109.6(5)$ & $\mathrm{N} 4-\mathrm{C} 10-\mathrm{C} 9$ & $110.3(2)$ \\
\hline $\mathrm{O}^{\prime}-\mathrm{Cl1}^{\prime}-\mathrm{O} 3^{\prime}$ & $110.8(5)$ & $\mathrm{N} 4-\mathrm{C} 10-\mathrm{H} 10 \mathrm{~A}$ & 109.6 \\
\hline $\mathrm{O} 6^{\prime}-\mathrm{Cl1} 1^{\prime}-\mathrm{O} 4^{\prime}$ & $108.9(5)$ & $\mathrm{C} 9-\mathrm{C} 10-\mathrm{H} 10 \mathrm{~A}$ & 109.6 \\
\hline $\mathrm{O} 5^{\prime}-\mathrm{Cl1}^{\prime}-\mathrm{O} 4^{\prime}$ & $110.2(5)$ & $\mathrm{N} 4-\mathrm{C} 10-\mathrm{H} 10 \mathrm{~B}$ & 109.6 \\
\hline $\mathrm{O} 3^{\prime}-\mathrm{Cl1}^{\prime}-\mathrm{O} 4^{\prime}$ & $110.8(5)$ & $\mathrm{C} 9-\mathrm{C} 10-\mathrm{H} 10 \mathrm{~B}$ & 109.6 \\
\hline $\mathrm{H} 11-\mathrm{O} 1 \mathrm{~W}-\mathrm{H} 12$ & $109.4(17)$ & $\mathrm{H} 10 \mathrm{~A}-\mathrm{C} 10-\mathrm{H} 10 \mathrm{~B}$ & 108.1 \\
\hline $\mathrm{C} 1-\mathrm{N} 1-\mathrm{C} 14$ & $113.35(19)$ & $\mathrm{N} 4-\mathrm{C} 11-\mathrm{C} 12$ & $112.5(2)$ \\
\hline $\mathrm{C} 1-\mathrm{N} 1-\mathrm{Ni} 1$ & $104.50(15)$ & $\mathrm{N} 4-\mathrm{C} 11-\mathrm{C} 13$ & $111.3(2)$ \\
\hline $\mathrm{C} 14-\mathrm{N} 1-\mathrm{Ni1}$ & $121.17(16)$ & $\mathrm{C} 12-\mathrm{C} 11-\mathrm{C} 13$ & $109.5(2)$ \\
\hline $\mathrm{C} 1-\mathrm{N} 1-\mathrm{H} 1$ & $101.7(19)$ & $\mathrm{N} 4-\mathrm{C} 11-\mathrm{H} 11 \mathrm{~A}$ & 107.8 \\
\hline $\mathrm{C} 14-\mathrm{N} 1-\mathrm{H} 1$ & $105.3(19)$ & $\mathrm{C} 12-\mathrm{C} 11-\mathrm{H} 11 \mathrm{~A}$ & 107.8 \\
\hline $\mathrm{Ni} 1-\mathrm{N} 1-\mathrm{H} 1$ & $109.1(18)$ & $\mathrm{C} 13-\mathrm{C} 11-\mathrm{H} 11 \mathrm{~A}$ & 107.8 \\
\hline $\mathrm{C} 2-\mathrm{N} 2-\mathrm{C} 3$ & $111.5(2)$ & $\mathrm{C} 11-\mathrm{C} 12-\mathrm{H} 12 \mathrm{~A}$ & 109.5 \\
\hline $\mathrm{C} 2-\mathrm{N} 2-\mathrm{Ni} 1$ & $105.70(15)$ & $\mathrm{C} 11-\mathrm{C} 12-\mathrm{H} 12 \mathrm{~B}$ & 109.5 \\
\hline $\mathrm{C} 3-\mathrm{N} 2-\mathrm{Ni} 1$ & $116.01(15)$ & $\mathrm{H} 12 \mathrm{~A}-\mathrm{C} 12-\mathrm{H} 12 \mathrm{~B}$ & 109.5 \\
\hline $\mathrm{C} 2-\mathrm{N} 2-\mathrm{H} 2$ & $106(2)$ & $\mathrm{C} 11-\mathrm{C} 12-\mathrm{H} 12 \mathrm{C}$ & 109.5 \\
\hline $\mathrm{C} 3-\mathrm{N} 2-\mathrm{H} 2$ & $110(2)$ & $\mathrm{H} 12 \mathrm{~A}-\mathrm{C} 12-\mathrm{H} 12 \mathrm{C}$ & 109.5 \\
\hline $\mathrm{Ni} 1-\mathrm{N} 2-\mathrm{H} 2$ & $107(2)$ & $\mathrm{H} 12 \mathrm{~B}-\mathrm{C} 12-\mathrm{H} 12 \mathrm{C}$ & 109.5 \\
\hline $\mathrm{C} 9-\mathrm{N} 3-\mathrm{C} 6$ & $114.5(2)$ & $\mathrm{C} 11-\mathrm{C} 13-\mathrm{C} 14$ & $117.97(19)$ \\
\hline $\mathrm{C} 9-\mathrm{N} 3-\mathrm{Ni} 1$ & $103.96(15)$ & $\mathrm{C} 11-\mathrm{C} 13-\mathrm{H} 13 \mathrm{~A}$ & 107.8 \\
\hline
\end{tabular}




\begin{tabular}{|c|c|c|c|}
\hline $\mathrm{C} 6-\mathrm{N} 3-\mathrm{Ni} 1$ & $121.14(16)$ & $\mathrm{C} 14-\mathrm{C} 13-\mathrm{H} 13 \mathrm{~A}$ & 107.8 \\
\hline $\mathrm{C} 9-\mathrm{N} 3-\mathrm{H} 3$ & $105(2)$ & $\mathrm{C} 11-\mathrm{C} 13-\mathrm{H} 13 \mathrm{~B}$ & 107.8 \\
\hline $\mathrm{C} 6-\mathrm{N} 3-\mathrm{H} 3$ & $107(2)$ & $\mathrm{C} 14-\mathrm{C} 13-\mathrm{H} 13 \mathrm{~B}$ & 107.8 \\
\hline $\mathrm{Ni} 1-\mathrm{N} 3-\mathrm{H} 3$ & $103(2)$ & $\mathrm{H} 13 \mathrm{~A}-\mathrm{C} 13-\mathrm{H} 13 \mathrm{~B}$ & 107.2 \\
\hline $\mathrm{C} 10-\mathrm{N} 4-\mathrm{C} 11$ & $112.8(2)$ & $\mathrm{N} 1-\mathrm{C} 14-\mathrm{C} 15$ & $107.37(19)$ \\
\hline $\mathrm{C} 10-\mathrm{N} 4-\mathrm{Ni} 1$ & $103.81(15)$ & $\mathrm{N} 1-\mathrm{C} 14-\mathrm{C} 16$ & $111.3(2)$ \\
\hline $\mathrm{C} 11-\mathrm{N} 4-\mathrm{Ni} 1$ & $114.50(15)$ & $\mathrm{C} 15-\mathrm{C} 14-\mathrm{C} 16$ & $107.4(2)$ \\
\hline $\mathrm{C} 10-\mathrm{N} 4-\mathrm{H} 4$ & $104(2)$ & $\mathrm{N} 1-\mathrm{C} 14-\mathrm{C} 13$ & $110.4(2)$ \\
\hline $\mathrm{C} 11-\mathrm{N} 4-\mathrm{H} 4$ & $109(2)$ & $\mathrm{C} 15-\mathrm{C} 14-\mathrm{C} 13$ & $111.2(2)$ \\
\hline $\mathrm{Ni} 1-\mathrm{N} 4-\mathrm{H} 4$ & $113(2)$ & $\mathrm{C} 16-\mathrm{C} 14-\mathrm{C} 13$ & $109.19(19)$ \\
\hline $\mathrm{C} 23-\mathrm{N} 5-\mathrm{H} 51$ & $119(2)$ & $\mathrm{C} 14-\mathrm{C} 15-\mathrm{H} 15 \mathrm{~A}$ & 109.5 \\
\hline $\mathrm{C} 23-\mathrm{N} 5-\mathrm{H} 52$ & $116(2)$ & $\mathrm{C} 14-\mathrm{C} 15-\mathrm{H} 15 \mathrm{~B}$ & 109.5 \\
\hline $\mathrm{H} 51-\mathrm{N} 5-\mathrm{H} 52$ & $121(3)$ & $\mathrm{H} 15 \mathrm{~A}-\mathrm{C} 15-\mathrm{H} 15 \mathrm{~B}$ & 109.5 \\
\hline $\mathrm{N} 1-\mathrm{C} 1-\mathrm{C} 2$ & $109.5(2)$ & $\mathrm{C} 14-\mathrm{C} 15-\mathrm{H} 15 \mathrm{C}$ & 109.5 \\
\hline $\mathrm{N} 1-\mathrm{C} 1-\mathrm{H} 1 \mathrm{~A}$ & 109.8 & $\mathrm{H} 15 \mathrm{~A}-\mathrm{C} 15-\mathrm{H} 15 \mathrm{C}$ & 109.5 \\
\hline $\mathrm{C} 2-\mathrm{C} 1-\mathrm{H} 1 \mathrm{~A}$ & 109.8 & $\mathrm{H} 15 \mathrm{~B}-\mathrm{C} 15-\mathrm{H} 15 \mathrm{C}$ & 109.5 \\
\hline $\mathrm{N} 1-\mathrm{C} 1-\mathrm{H} 1 \mathrm{~B}$ & 109.8 & $\mathrm{C} 14-\mathrm{C} 16-\mathrm{H} 16 \mathrm{~A}$ & 109.5 \\
\hline $\mathrm{C} 2-\mathrm{C} 1-\mathrm{H} 1 \mathrm{~B}$ & 109.8 & $\mathrm{C} 14-\mathrm{C} 16-\mathrm{H} 16 \mathrm{~B}$ & 109.5 \\
\hline $\mathrm{H} 1 \mathrm{~A}-\mathrm{C} 1-\mathrm{H} 1 \mathrm{~B}$ & 108.2 & $\mathrm{H} 16 \mathrm{~A}-\mathrm{C} 16-\mathrm{H} 16 \mathrm{~B}$ & 109.5 \\
\hline $\mathrm{N} 2-\mathrm{C} 2-\mathrm{C} 1$ & $109.3(2)$ & $\mathrm{C} 14-\mathrm{C} 16-\mathrm{H} 16 \mathrm{C}$ & 109.5 \\
\hline $\mathrm{N} 2-\mathrm{C} 2-\mathrm{H} 2 \mathrm{~A}$ & 109.8 & $\mathrm{H} 16 \mathrm{~A}-\mathrm{C} 16-\mathrm{H} 16 \mathrm{C}$ & 109.5 \\
\hline $\mathrm{C} 1-\mathrm{C} 2-\mathrm{H} 2 \mathrm{~A}$ & 109.8 & $\mathrm{H} 16 \mathrm{~B}-\mathrm{C} 16-\mathrm{H} 16 \mathrm{C}$ & 109.5 \\
\hline $\mathrm{N} 2-\mathrm{C} 2-\mathrm{H} 2 \mathrm{~B}$ & 109.8 & $\mathrm{O} 2-\mathrm{C} 17-\mathrm{O} 1$ & $119.3(2)$ \\
\hline $\mathrm{C} 1-\mathrm{C} 2-\mathrm{H} 2 \mathrm{~B}$ & 109.8 & $\mathrm{O} 2-\mathrm{C} 17-\mathrm{C} 18$ & $120.9(2)$ \\
\hline $\mathrm{H} 2 \mathrm{~A}-\mathrm{C} 2-\mathrm{H} 2 \mathrm{~B}$ & 108.3 & $\mathrm{O} 1-\mathrm{C} 17-\mathrm{C} 18$ & $119.8(2)$ \\
\hline $\mathrm{N} 2-\mathrm{C} 3-\mathrm{C} 5$ & $110.5(2)$ & $\mathrm{C} 19-\mathrm{C} 18-\mathrm{C} 23$ & $119.9(2)$ \\
\hline $\mathrm{N} 2-\mathrm{C} 3-\mathrm{C} 4$ & $111.9(2)$ & $\mathrm{C} 19-\mathrm{C} 18-\mathrm{C} 17$ & $118.9(2)$ \\
\hline $\mathrm{C} 5-\mathrm{C} 3-\mathrm{C} 4$ & $110.0(2)$ & $\mathrm{C} 23-\mathrm{C} 18-\mathrm{C} 17$ & $121.3(2)$ \\
\hline $\mathrm{N} 2-\mathrm{C} 3-\mathrm{H} 3 \mathrm{a}$ & 108.1 & $\mathrm{C} 20-\mathrm{C} 19-\mathrm{C} 18$ & $121.8(2)$ \\
\hline $\mathrm{C} 5-\mathrm{C} 3-\mathrm{H} 3 \mathrm{a}$ & 108.1 & $\mathrm{C} 20-\mathrm{C} 19-\mathrm{H} 19$ & 119.1 \\
\hline $\mathrm{C} 4-\mathrm{C} 3-\mathrm{H} 3 \mathrm{a}$ & 108.1 & $\mathrm{C} 18-\mathrm{C} 19-\mathrm{H} 19$ & 119.1 \\
\hline $\mathrm{C} 3-\mathrm{C} 4-\mathrm{H} 4 \mathrm{~A}$ & 109.5 & $\mathrm{C} 19-\mathrm{C} 20-\mathrm{C} 21$ & $118.6(2)$ \\
\hline $\mathrm{C} 3-\mathrm{C} 4-\mathrm{H} 4 \mathrm{~B}$ & 109.5 & $\mathrm{C} 19-\mathrm{C} 20-\mathrm{H} 20$ & 120.7 \\
\hline $\mathrm{H} 4 \mathrm{~A}-\mathrm{C} 4-\mathrm{H} 4 \mathrm{~B}$ & 109.5 & $\mathrm{C} 21-\mathrm{C} 20-\mathrm{H} 20$ & 120.7 \\
\hline $\mathrm{C} 3-\mathrm{C} 4-\mathrm{H} 4 \mathrm{C}$ & 109.5 & $\mathrm{C} 22-\mathrm{C} 21-\mathrm{C} 20$ & $120.7(2)$ \\
\hline $\mathrm{H} 4 \mathrm{~A}-\mathrm{C} 4-\mathrm{H} 4 \mathrm{C}$ & 109.5 & $\mathrm{C} 22-\mathrm{C} 21-\mathrm{H} 21$ & 119.6 \\
\hline $\mathrm{H} 4 \mathrm{~B}-\mathrm{C} 4-\mathrm{H} 4 \mathrm{C}$ & 109.5 & $\mathrm{C} 20-\mathrm{C} 21-\mathrm{H} 21$ & 119.6 \\
\hline $\mathrm{C} 3-\mathrm{C} 5-\mathrm{C} 6$ & $118.7(2)$ & $\mathrm{C} 21-\mathrm{C} 22-\mathrm{C} 23$ & $121.7(3)$ \\
\hline $\mathrm{C} 3-\mathrm{C} 5-\mathrm{H} 5 \mathrm{~A}$ & 107.6 & $\mathrm{C} 21-\mathrm{C} 22-\mathrm{H} 22$ & 119.1 \\
\hline $\mathrm{C} 6-\mathrm{C} 5-\mathrm{H} 5 \mathrm{~A}$ & 107.6 & $\mathrm{C} 23-\mathrm{C} 22-\mathrm{H} 22$ & 119.1 \\
\hline $\mathrm{C} 3-\mathrm{C} 5-\mathrm{H} 5 \mathrm{~B}$ & 107.6 & $\mathrm{~N} 5-\mathrm{C} 23-\mathrm{C} 18$ & $123.1(2)$ \\
\hline $\mathrm{C} 6-\mathrm{C} 5-\mathrm{H} 5 \mathrm{~B}$ & 107.6 & $\mathrm{~N} 5-\mathrm{C} 23-\mathrm{C} 22$ & $119.6(2)$ \\
\hline $\mathrm{H} 5 \mathrm{~A}-\mathrm{C} 5-\mathrm{H} 5 \mathrm{~B}$ & 107.1 & $\mathrm{C} 18-\mathrm{C} 23-\mathrm{C} 22$ & $117.3(2)$ \\
\hline $\mathrm{N} 2-\mathrm{Ni} 1-\mathrm{O} 1-\mathrm{C} 17$ & $-31.3(3)$ & $\mathrm{C} 2-\mathrm{N} 2-\mathrm{C} 3-\mathrm{C} 4$ & $51.2(3)$ \\
\hline $\mathrm{N} 4-\mathrm{Ni1}-\mathrm{O} 1-\mathrm{C} 17$ & $171.28(14)$ & $\mathrm{Ni} 1-\mathrm{N} 2-\mathrm{C} 3-\mathrm{C} 4$ & $172.23(19)$ \\
\hline $\mathrm{N} 1-\mathrm{Ni1}-\mathrm{O} 1-\mathrm{C} 17$ & $79.59(14)$ & $\mathrm{N} 2-\mathrm{C} 3-\mathrm{C} 5-\mathrm{C} 6$ & $71.2(3)$ \\
\hline $\mathrm{O} 2-\mathrm{Ni} 1-\mathrm{O} 1-\mathrm{C} 17$ & 0.00 & $\mathrm{C} 4-\mathrm{C} 3-\mathrm{C} 5-\mathrm{C} 6$ & $-164.7(2)$ \\
\hline
\end{tabular}




$$
\begin{aligned}
& \text { N3-Ni1-O1-C17 } \\
& \text { N2-Ni1-O2-C17 } \\
& \text { N4-Ni1-O2-C17 } \\
& \text { N1-Ni1-O2-C17 } \\
& \text { N3-Ni1-O2-C17 } \\
& \text { O1-Ni1-O2-C17 } \\
& \text { N2-Ni1-N1-C1 } \\
& \text { N4-Ni1-N1-C1 } \\
& \text { O2-Ni1-N1-C1 } \\
& \text { O1-Ni1-N1-C1 } \\
& \text { N2-Ni1-N1-C14 } \\
& \text { N4-Ni1-N1-C14 } \\
& \text { O2-Ni1-N1-C14 } \\
& \text { O1-Ni1-N1-C14 } \\
& \text { N4-Ni1-N2-C2 } \\
& \text { N1-Ni1-N2-C2 } \\
& \text { O2-Ni1-N2-C2 } \\
& \text { N3-Ni1-N2-C2 } \\
& \text { O1-Ni1-N2-C2 } \\
& \text { N4-Ni1-N2-C3 } \\
& \text { N1-Ni1-N2-C3 } \\
& \text { O2-Ni1-N2-C3 } \\
& \text { N3-Ni1-N2-C3 } \\
& \text { O1-Ni1-N2-C3 } \\
& \text { N2-Ni1-N3-C9 } \\
& \text { N4-Ni1-N3-C9 } \\
& \text { O2-Ni1-N3-C9 } \\
& \text { O1-Ni1-N3-C9 } \\
& \text { N2-Ni1-N3-C6 } \\
& \text { N4-Ni1-N3-C6 } \\
& \text { O2-Ni1-N3-C6 } \\
& \text { O1-Ni1-N3-C6 } \\
& \text { N2-Ni1-N4-C10 } \\
& \text { N1-Ni1-N4-C10 } \\
& \text { O2-Ni1-N4-C10 } \\
& \text { N3-Ni1-N4-C10 } \\
& \text { O1-Ni1-N4-C10 } \\
& \text { N2-Ni1-N4-C11 } \\
& \text { N1-Ni1-N4-C11 } \\
& \text { O2-Ni1-N4-C11 } \\
& \text { N3-Ni1-N4-C11 } \\
& \text { O1-Ni1-N4-C11 } \\
& \text { C14-N1-C1-C2 } \\
& \text { Ni1-N1-C1-C2 } \\
& \text { C3-N2-C2-C1 } \\
& \text { Ni1-N2-C2-C1 } \\
& \text { N1-C1-C2-N2 } \\
& \text { C2-N2-C3-C5 }
\end{aligned}
$$

\begin{tabular}{|c|c|}
\hline $\mathrm{C} 9-\mathrm{N} 3-\mathrm{C} 6-\mathrm{C} 7$ & $163.1(2)$ \\
\hline $\mathrm{Ni} 1-\mathrm{N} 3-\mathrm{C} 6-\mathrm{C} 7$ & $-71.0(2)$ \\
\hline $\mathrm{C} 9-\mathrm{N} 3-\mathrm{C} 6-\mathrm{C} 5$ & $-76.4(3)$ \\
\hline $\mathrm{Ni} 1-\mathrm{N} 3-\mathrm{C} 6-\mathrm{C} 5$ & $49.4(3)$ \\
\hline $\mathrm{C} 9-\mathrm{N} 3-\mathrm{C} 6-\mathrm{C} 8$ & $44.6(3)$ \\
\hline $\mathrm{Ni} 1-\mathrm{N} 3-\mathrm{C} 6-\mathrm{C} 8$ & $170.46(18)$ \\
\hline $\mathrm{C} 3-\mathrm{C} 5-\mathrm{C} 6-\mathrm{N} 3$ & $-61.6(3)$ \\
\hline $\mathrm{C} 3-\mathrm{C} 5-\mathrm{C} 6-\mathrm{C} 7$ & $56.8(3)$ \\
\hline $\mathrm{C} 3-\mathrm{C} 5-\mathrm{C} 6-\mathrm{C} 8$ & $175.3(2)$ \\
\hline $\mathrm{C} 6-\mathrm{N} 3-\mathrm{C} 9-\mathrm{C} 10$ & $174.1(2)$ \\
\hline $\mathrm{Ni1}-\mathrm{N} 3-\mathrm{C} 9-\mathrm{C} 10$ & $39.7(2)$ \\
\hline $\mathrm{C} 11-\mathrm{N} 4-\mathrm{C} 10-\mathrm{C} 9$ & $169.1(2)$ \\
\hline $\mathrm{Ni1}-\mathrm{N} 4-\mathrm{C} 10-\mathrm{C} 9$ & $44.6(2)$ \\
\hline $\mathrm{N} 3-\mathrm{C} 9-\mathrm{C} 10-\mathrm{N} 4$ & $-59.9(3)$ \\
\hline $\mathrm{C} 10-\mathrm{N} 4-\mathrm{C} 11-\mathrm{C} 12$ & $53.3(3)$ \\
\hline $\mathrm{Ni1}-\mathrm{N} 4-\mathrm{C} 11-\mathrm{C} 12$ & $171.73(16)$ \\
\hline $\mathrm{C} 10-\mathrm{N} 4-\mathrm{C} 11-\mathrm{C} 13$ & $176.57(19)$ \\
\hline $\mathrm{Ni1}-\mathrm{N} 4-\mathrm{C} 11-\mathrm{C} 13$ & $-65.0(2)$ \\
\hline $\mathrm{N} 4-\mathrm{C} 11-\mathrm{C} 13-\mathrm{C} 14$ & $71.8(3)$ \\
\hline $\mathrm{C} 12-\mathrm{C} 11-\mathrm{C} 13-\mathrm{C} 14$ & $-163.2(2)$ \\
\hline $\mathrm{C} 1-\mathrm{N} 1-\mathrm{C} 14-\mathrm{C} 15$ & $163.3(2)$ \\
\hline $\mathrm{Ni1}-\mathrm{N} 1-\mathrm{C} 14-\mathrm{C} 15$ & $-71.2(2)$ \\
\hline $\mathrm{C} 1-\mathrm{N} 1-\mathrm{C} 14-\mathrm{C} 16$ & $46.1(3)$ \\
\hline $\mathrm{Ni1}-\mathrm{N} 1-\mathrm{C} 14-\mathrm{C} 16$ & $171.49(16)$ \\
\hline $\mathrm{C} 1-\mathrm{N} 1-\mathrm{C} 14-\mathrm{C} 13$ & $-75.4(2)$ \\
\hline $\mathrm{Ni1}-\mathrm{N} 1-\mathrm{C} 14-\mathrm{C} 13$ & $50.1(2)$ \\
\hline $\mathrm{C} 11-\mathrm{C} 13-\mathrm{C} 14-\mathrm{N} 1$ & $-61.3(3)$ \\
\hline $\mathrm{C} 11-\mathrm{C} 13-\mathrm{C} 14-\mathrm{C} 15$ & $57.7(3)$ \\
\hline $\mathrm{C} 11-\mathrm{C} 13-\mathrm{C} 14-\mathrm{C} 16$ & $176.0(2)$ \\
\hline $\mathrm{Ni} 1-\mathrm{O} 2-\mathrm{C} 17-\mathrm{O} 1$ & $0.0(2)$ \\
\hline $\mathrm{Ni} 1-\mathrm{O} 2-\mathrm{C} 17-\mathrm{C} 18$ & $179.87(19)$ \\
\hline $\mathrm{Ni1}-\mathrm{O} 1-\mathrm{C} 17-\mathrm{O} 2$ & $0.0(2)$ \\
\hline $\mathrm{Ni} 1-\mathrm{O} 1-\mathrm{C} 17-\mathrm{C} 18$ & $-179.88(19$ \\
\hline $\mathrm{O} 2-\mathrm{C} 17-\mathrm{C} 18-\mathrm{C} 19$ & $155.0(2)$ \\
\hline $\mathrm{O} 1-\mathrm{C} 17-\mathrm{C} 18-\mathrm{C} 19$ & $-25.2(3)$ \\
\hline $\mathrm{O} 2-\mathrm{C} 17-\mathrm{C} 18-\mathrm{C} 23$ & $-26.9(3)$ \\
\hline $\mathrm{O} 1-\mathrm{C} 17-\mathrm{C} 18-\mathrm{C} 23$ & $152.9(2)$ \\
\hline $\mathrm{C} 23-\mathrm{C} 18-\mathrm{C} 19-\mathrm{C} 20$ & $0.8(4)$ \\
\hline $\mathrm{C} 17-\mathrm{C} 18-\mathrm{C} 19-\mathrm{C} 20$ & $178.9(2)$ \\
\hline $\mathrm{C} 18-\mathrm{C} 19-\mathrm{C} 20-\mathrm{C} 21$ & $-2.2(4)$ \\
\hline $\mathrm{C} 19-\mathrm{C} 20-\mathrm{C} 21-\mathrm{C} 22$ & $1.7(4)$ \\
\hline $\mathrm{C} 20-\mathrm{C} 21-\mathrm{C} 22-\mathrm{C} 23$ & $0.3(4)$ \\
\hline $\mathrm{C} 19-\mathrm{C} 18-\mathrm{C} 23-\mathrm{N} 5$ & $-178.1(2)$ \\
\hline $\mathrm{C} 17-\mathrm{C} 18-\mathrm{C} 23-\mathrm{N} 5$ & $3.8(4)$ \\
\hline $\mathrm{C} 19-\mathrm{C} 18-\mathrm{C} 23-\mathrm{C} 22$ & $1.1(3)$ \\
\hline $\mathrm{C} 17-\mathrm{C} 18-\mathrm{C} 23-\mathrm{C} 22$ & $-176.9(2)$ \\
\hline $\mathrm{C} 21-\mathrm{C} 22-\mathrm{C} 23-\mathrm{N} 5$ & $177.6(2)$ \\
\hline $\mathrm{C} 21-\mathrm{C} 22-\mathrm{C} 23-\mathrm{C} 18$ & $-1.7(4)$ \\
\hline
\end{tabular}

$-103.62(14)$
$169.89(14)$
$-21.1(2)$
$-105.97(14)$
$78.28(15)$
$0.00(13)$
$-14.19(16)$
$88.57(16)$
$-116.17(16)$
$-176.11(15)$
$-143.61(18)$
$-40.84(18)$
$114.42(17)$
$54.48(18)$
$-104.54(16)$
$-15.15(16)$
$70.77(16)$
$169.98(16)$
$98.6(2)$
$131.31(17)$
$-139.30(18)$
$-53.38(18)$
$45.83(18)$
$-25.6(3)$
$90.51(16)$
$-12.31(16)$
$-167.74(16)$
$-107.87(16)$
$-40.04(18)$
$-142.86(18)$
$61.70(18)$
$121.58(18)$
$-105.88(17)$
$169.26(17)$
$85.1(2)$
$-17.14(17)$
$66.63(17)$
$130.71(15)$
$45.86(16)$
$-38.3(3)$
$-140.55(16)$
$-56.77(16)$
$175.3(2)$
$41.4(2)$
$169.3(2)$
$42.4(2)$
$-58.6(3)$
$174.1(2)$

$163.1(2)$

$-76.4(3)$

$49.4(3)$

$44.6(3)$

$170.46(18)$

$-61.6(3)$

$175.3(2)$

(2)

$169.1(2)$

$44.6(2)$

$-59.9(3)$

$171.73(16)$

$176.57(19)$

$-65.0(2)$

$71.8(3)$

$63.3(2)$

$-71.2(2)$

$6.1(3)$

$-75.4(2)$

$50.1(2)$

$-61.3(3)$

57.7 (3)

$176.0(2)$

$0.0(2)$

$179.87(19)$

$-179.88(19)$

$55.0(2)$

$25.2(3)$

$0.8(4)$

$178.9(2)$

$-2.2(4)$

$1.7(4)$

$0.3(4)$

$3.8(4)$

1.1 (3)

$177.6(2)$ 
$\mathrm{Ni1}-\mathrm{N} 2-\mathrm{C} 3-\mathrm{C} 5 \quad-64.8(2)$

Hydrogen-bond geometry $\left(\AA,{ }^{\circ}\right)$

\begin{tabular}{lllll}
\hline$D-\mathrm{H} \cdots A$ & $D-\mathrm{H}$ & $\mathrm{H} \cdots A$ & $D \cdots A$ & $D-\mathrm{H} \cdots A$ \\
\hline $\mathrm{N} 1-\mathrm{H} 1 \cdots \mathrm{O}^{2} w^{\mathrm{i}}$ & $0.86(2)$ & $2.15(2)$ & $2.995(3)$ & $166(2)$ \\
$\mathrm{N} 2-\mathrm{H} 2 \cdots 5^{\mathrm{ii}}$ & $0.86(2)$ & $2.56(2)$ & $3.304(8)$ & $147(2)$ \\
$\mathrm{N} 2-\mathrm{H} 2 \cdots \mathrm{O} 5^{\prime i \mathrm{ii}}$ & $0.86(2)$ & $2.35(2)$ & $3.125(11)$ & $150(3)$ \\
$\mathrm{N} 3-\mathrm{H} 3 \cdots \mathrm{O} 1$ & $0.86(2)$ & $2.49(3)$ & $2.885(3)$ & $109(2)$ \\
$\mathrm{N} 4-\mathrm{H} 4 \cdots 6^{\mathrm{ii}}$ & $0.86(3)$ & $2.61(2)$ & $3.343(6)$ & $145(3)$ \\
$\mathrm{N} 4-\mathrm{H} 4 \cdots 6^{\prime i i}$ & $0.86(3)$ & $2.40(2)$ & $3.195(6)$ & $155(3)$ \\
$\mathrm{N} 5-\mathrm{H} 51 \cdots \mathrm{O} 2$ & $0.85(3)$ & $2.13(3)$ & $2.751(3)$ & $129(2)$ \\
$\mathrm{N} 5-\mathrm{H} 52 \cdots \mathrm{O} 4^{\prime i}$ & $0.85(1)$ & $2.32(1)$ & $3.155(6)$ & $168(3)$ \\
$\mathrm{O} 1 \mathrm{w}-\mathrm{H} 11 \cdots \mathrm{O} 1$ & $0.84(2)$ & $1.96(2)$ & $2.795(2)$ & $172(3)$ \\
$\mathrm{O} 1 \mathrm{w}-\mathrm{H} 12 \cdots \mathrm{O} 3$ & $0.83(2)$ & $2.26(2)$ & $3.077(9)$ & $167(3)$ \\
$\mathrm{O} 1 \mathrm{w}-\mathrm{H} 12 \cdots \mathrm{O}^{\prime}$ & $0.83(2)$ & $2.09(2)$ & $2.890(9)$ & $160(3)$
\end{tabular}

Symmetry codes: (i) $x,-y+1, z+1 / 2$; (ii) $x-1 / 2,-y+1 / 2, z+1 / 2$. 